\title{
RELATIVE RELATION MODULES OF FINITE GROUPS
}

\author{
by MOHAMMAD YAMIN
}

(Received 4th January 1990)

\begin{abstract}
Let $E$ be a free product of a finite number of cyclic groups, and $S$ a normal subgroup of $E$ such that $E / S \cong G$ is finite. For a prime $p, \hat{S}=S / S^{\prime} S^{p}$ may be regarded as an $\mathbb{F}_{p} G$-module. Whenever $E$ is a free group, $\hat{S}$ is called a relation module (modulo $p$ ); in general we call $\hat{S}$ a relative relation module (modulo $p$ ). Gaschütz, Gruenberg and others have studied relation modules; the aim of this paper is to study relative relation modules.
\end{abstract}

1980 Mathematics subject classification (1985 Revision): 16A26 and $20 \mathrm{C05}$.

\section{Introduction}

Throughout the paper, let $p$ be a (fixed) prime, $\mathbb{F}_{p}$ the field of $p$ elements, $G$ a finite group of order $n$ generated by $X=\left\{g_{i}, 1 \leqq i \leqq d\right\}, G_{i}$ the cyclic subgroup of $G$ of order $n_{i}$ generated by $g_{i}$ and $E_{i}$ a cyclic group of order $m_{i}$, where $m_{i}=k_{i} n_{i}, 1 \leqq k_{i} \leqq \infty$. Suppose that $k_{i}<\infty$ and $p \nmid k_{i}$ if $i \leqq \delta, \delta \leqq d$, and $k_{i}=\infty$ or $p \mid k_{i}$ if $\delta+1 \leqq i \leqq d$. Let $E$ be the free product of the $E_{i}, 1 \leqq i \leqq d$; and $S$ the kernel of the natural epimorphism of $E$ onto $G$.

$\hat{S}=S / S^{\prime} S^{p}$, regarded as an $\mathbb{F}_{p} G$-module via conjugation in $E$, is called a relation module (modulo $p$ ) when $E$ is a free group. In general $\hat{S}$ will be called a relative relation module (For convenience, reference to $p$ is being dropped). We remark that our notion of relative relation module is different from that discussed in [5]. Gaschütz [2]; Gruenberg [3] and [4], and others have studied relation modules. In this paper, along the lines of Gruenberg's theory of relation modules, we study exact sequences, decomposition and comparison of relative relation modules.

Crucial to our study is the relationship between relation and relative relation modules, which enables us to establish an exact sequence: $\hat{S}$ is embedded in the direct sum of the augmentation ideals of the $\mathbb{F}_{p} G_{i}$ induced to $G, 1 \leqq i \leqq \delta$, and a free module of rank $d-\delta$; the resulting factor module is isomorphic to the augmentation ideal of $\mathbb{F}_{p} G$. Another exact sequence is established in which $\hat{S}$, when $\delta \geqq 1$, is embedded in a free module of rank $d-1$. These exact sequences are established in Section 2 .

In Section 3 we discuss the structure of $\hat{S}$. When $p$ is coprime to $|G|$, relative relation modules are easily described; our attempts in the non-coprime case have been only partially successful however. Whenever $p|| G \mid$ but $p \nmid\left|G_{i}\right|, 1 \leqq i \leqq \delta$; we say that $p$ is semi-coprime to $|G|$ (with respect to $X$ ). In the coprime and semi-coprime cases, a characterisation, including a criterion for counting projective summands, of $\hat{S}$ is given. 
Given a decomposition $\hat{S} \cong P(\hat{S}) \oplus N(\hat{S})$, where $P(\hat{S})$ is $\mathbb{F}_{p} G$-projective and $N(\hat{S})$ contains no projective summand, $P(\hat{S})$ is called the projective part and $N(\hat{S})$ the non-projective part of $\hat{S}$. In the semi-coprime case, $N(\hat{S})$ is nonzero and indecomposable (and also isomorphic to $N(\hat{S})$ of the case when $E$ is a free group). Nothing is predictable in the non semi-coprime case, $N(\hat{S})$ may be zero or decomposable (and may not even be a homomorphic image of $N(\hat{S})$ of the case when $E$ is a free group). The author [9] has shown that $\hat{S}$ is non-projective and indecomposable in the case when $G$ is a p-group, $X$ is a minimal generating set of $G$ and $\delta=d$.

In Section 4 we compare various relative relation modules. Two relative relation modules, isomorphic as $\mathbb{F}_{p}$-spaces, are rarely isomorphic as $G$-modules; that is, $\hat{S}$ not only depends on $G, p$ and $d$ but also on the mapping of $E$ to $G$. In cases when one relative relation module is embedded into another it does not necessarily mean that the bigger module splits over the smaller; however there are some known cases when it does happen.

\section{Relative relation modules and associated exact sequences}

Let $F$ be a free group freely generated by $\left\{f_{i}, 1 \leqq i \leqq d\right\}$ and consider

$$
1 \rightarrow R \rightarrow F \stackrel{\pi}{\rightarrow} G \rightarrow 1
$$

and

$$
1 \rightarrow S \rightarrow E \stackrel{\psi}{\rightarrow} G \rightarrow 1
$$

where $f_{i} \pi=g_{i}=e_{i} \psi, 1 \leqq i \leqq d$. Let $\hat{R}$ and $\hat{S}$ be the corresponding relation and relative relation modules of $G$ determined by $\pi$ and $\psi$.

The natural epimorphism $\phi: F \rightarrow E$ defined by $f_{i} \phi=e_{i}, 1 \leqq i \leqq d$, induces an epimorphism $\hat{\phi}: F / R^{\prime} R^{p} \rightarrow E / S^{\prime} S^{p}$ such that $\left(f_{i} R^{\prime} R^{p}\right) \hat{\phi}=e_{i} S^{\prime} S^{p}, 1 \leqq i \leqq \hat{d}$. If $\hat{Q}$ denotes the kernel of $\hat{\phi}$, and $\hat{\theta}$ the restriction of $\hat{\phi}$ to $\hat{R}$, then it may be checked that

$$
0 \rightarrow \hat{Q} \rightarrow \hat{R} \stackrel{\hat{\theta}}{\rightarrow} \hat{S} \rightarrow 0
$$

is $\mathbb{F}_{p} G$-exact, and that

$$
\widehat{Q}=\left\langle\left(f^{-1} f_{i}^{m_{i}} f\right) R^{\prime} R^{p} ; f \in F, 1 \leqq i \leqq \delta\right\rangle
$$

Let $B=\bigoplus_{i=1}^{d} b_{i} \mathbb{F}_{p} G$, a free module with $\left\{b_{i}, 1 \leqq i \leqq d\right\}$ as an $\mathbb{F}_{p} G$ basis. By the embedding 
theorem of Magnus [5], $F / R^{\prime} R^{p}$ may be embedded into the semidirect product of $B$ and $G$ such that $f_{i} R^{\prime} R^{p} \rightarrow\left(g_{i}, b_{i}\right), 1 \leqq i \leqq d$. This together with (2.3) gives:

Lemma 2.4. Let $T_{i}$ be the trivial irreducible submodule of $b_{i} \mathbb{F}_{p} G_{i}$ generated by $t_{i}=b_{i}\left(1+g_{i}+g_{i}^{2}+\cdots+g_{i}^{n_{i}-1}\right)$. Then

$$
0 \rightarrow \bigoplus_{i=1}^{\delta} T_{i}^{G} \rightarrow \hat{R} \stackrel{\hat{\theta}}{\rightarrow} \hat{S} \rightarrow 0
$$

where $\hat{\theta}$ is as defined above, is exact, and $\hat{Q}=\bigoplus_{i=1}^{\delta} T_{i}^{G}$.

Corollary 2.6.

$$
\operatorname{dim}_{F_{p}} \hat{S}=n(d-1)-\left(\sum_{i=1}^{\delta} \frac{n}{n_{i}}\right)+1
$$

Proof. By O. Schreier the rank of the free group $R$ is equal to $n(d-1)+1$, which is also equal to the dimension of $\hat{R}$. As $\operatorname{dim} T_{i}^{G}=\left(n / n_{i}\right)$, the result follows from (2.5).

Note 2.7. If $m_{i}=n_{i}$ for $1 \leqq i \leqq \delta$, and $m_{i}=\infty$ for $\delta+1 \leqq i \leqq d$, then by the Kurosh subgroup theorem ([7, Cor. 4.9.1, p. 243]) $S$ is a free group. In this case the rank of $S$ equals the dimension of $\hat{S}$ given by Corollary 2.6 .

Proposition 2.8. (Gaschütz [2]). Given the free presentation (2.1), then

$$
0 \rightarrow \hat{R} \rightarrow \bigoplus_{i=1}^{d} b_{i} \mathbb{F}_{p} G \stackrel{\hat{n}}{\rightarrow} \mathbf{g} \rightarrow 0
$$

is $\mathbb{F}_{p} G$-exact, where $\mathrm{g}$ denotes the augmentation ideal of $\mathbb{F}_{p} G$, and $\hat{\pi}$ is determined by $b_{i} \rightarrow g_{i}-1,1 \leqq i \leqq d$.

The sequence (2.9) is called the relation sequence (modulo $p$ ) of $G$ determined by (2.1). We have:

Proposition 2.10. Given (2.2) then the sequence, which we will call the relative relation sequence, 


$$
0 \rightarrow \widehat{S} \rightarrow \bigoplus_{i=1}^{\delta} \mathbf{g}_{i}^{G} \bigoplus_{i=\delta+1}^{d} b_{i} \mathbb{F}_{p} G \stackrel{\Downarrow}{\rightarrow} \mathbf{g} \rightarrow 0
$$

is $\mathbb{F}_{p} G$-exact, where $g_{i}$ denotes the augmentation ideal of $b_{i} \mathbb{F}_{p} G_{i}$, and $\hat{\psi}$ is determined by

$$
\begin{gathered}
b_{i}\left(g_{i}-1\right) \rightarrow g_{i}-1, \quad 1 \leqq i \leqq \delta \\
b_{i} \rightarrow g_{i}-1, \quad \delta+1 \leqq i \leqq d .
\end{gathered}
$$

Proof. The result follows by an application of the $3 \times 3$ lemma to the diagram

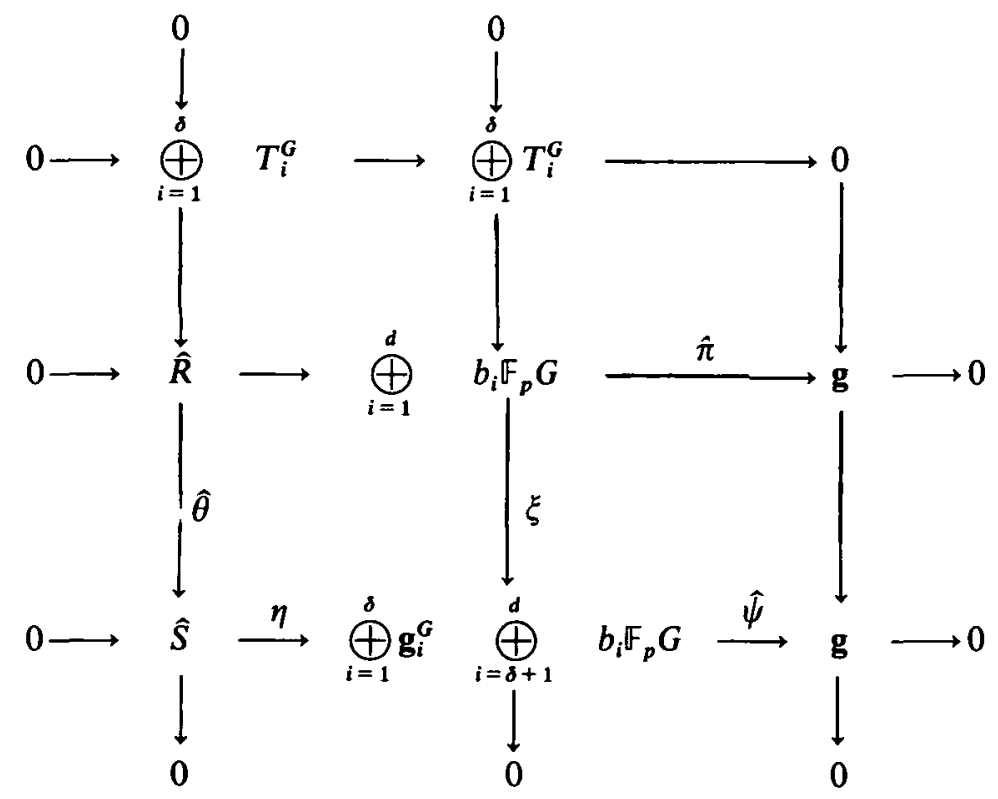

where

$$
\left(\sum_{i=1}^{d} b_{i} x_{i}\right) \xi=\sum_{i=1}^{\delta} b_{i}\left(g_{i}-1\right) x_{i}+\sum_{i=\delta+1}^{d} b_{i} x_{i}, \quad x_{i} \in \mathbb{F}_{p} G
$$

Next suppose that $\delta \geqq 1$, consider the diagram 


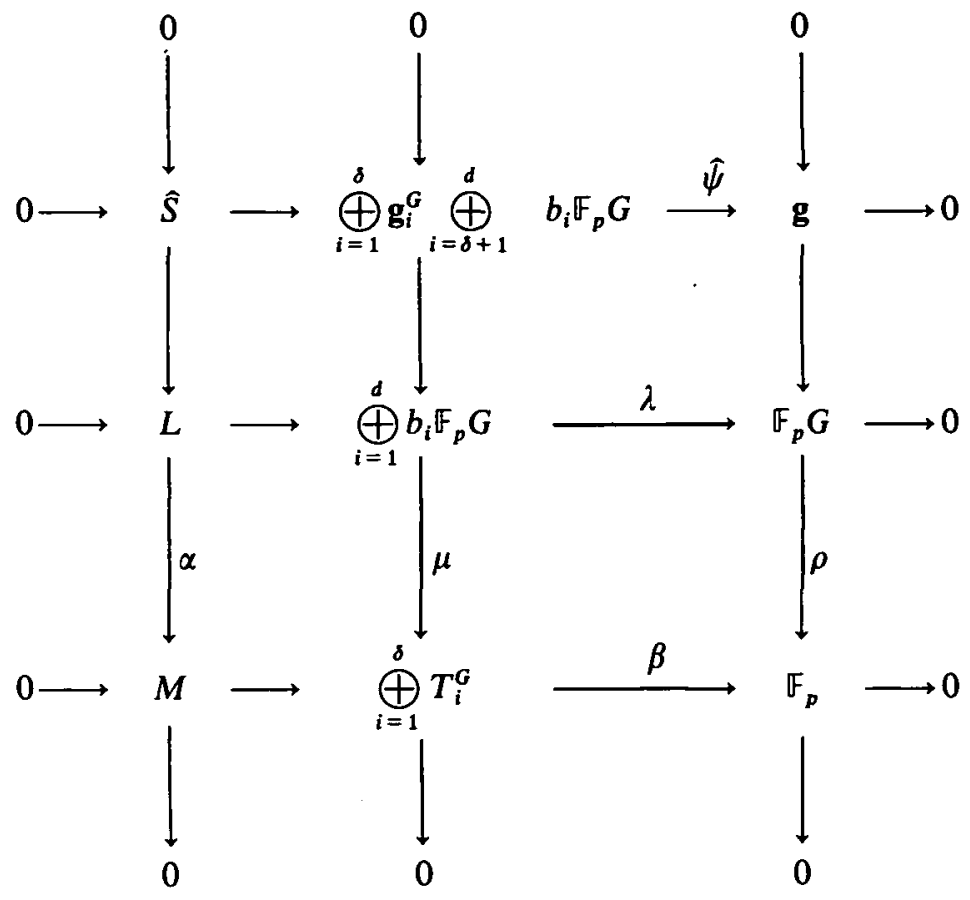

where

$$
\begin{gathered}
\left(\sum_{i=1}^{d} b_{i} x_{i}\right) \mu=\sum_{i=1}^{\delta} t_{i} x_{i}, \quad x_{i} \in \mathbb{F}_{p} G, \\
\left(\sum_{i=1}^{d} b_{i} x_{i}\right) \lambda=\sum_{i=1}^{\delta} x_{i}+\sum_{i=\delta+1}^{d}\left(g_{i}-1\right) x_{i}, \quad x_{i} \in \mathbb{F}_{p} G .
\end{gathered}
$$

Let $L$ be the kernel of $\lambda, \rho$ the augmentation, $M$ the kernel of $\beta$ and $\alpha$ the restriction of $\mu$ to $L$. The $3 \times 3$ lemma yields:

Proposition 2.12. Suppose that $\delta \geqq 1$, and let $L$ be a free module of rank $d-1$. Then

$$
0 \rightarrow \hat{S} \rightarrow L \stackrel{\alpha}{\rightarrow} \stackrel{\delta}{\oplus}_{i=1}^{G} T_{i}^{G} \stackrel{\beta}{\rightarrow} \mathbb{F}_{p} \rightarrow 0
$$

is $\mathbb{F}_{p} G$-exact, where $\alpha$ and $\beta$ are as described above.

It may be noted that, if $\delta=0, \hat{S}$ being a relation module cannot be embedded into a free module of rank $d-1$. 


\section{Structure of relative relation modules}

An application of Maschke's Theorem to the relative relation sequence (2.11) yields:

Proposition 3.1. Suppose that $p \nmid n$. Then

$$
\hat{S} \oplus \mathbf{g} \cong \bigoplus_{i=1}^{\delta} \mathbf{g}_{i}^{G} \bigoplus_{i=\delta+1}^{d} b_{i} \mathbb{F}_{p} G
$$

Since $\mathbf{g}$ and $\mathbf{g}_{i}^{G}$ contain no trivial submodules in the coprime case, $\hat{S}$ contains exactly $d-\delta$ copies of the trivial module $\mathbb{F}_{p}$.

Both $T_{i}^{G}$ and $\mathbf{g}_{i}^{G}$ are projective, precisely when $p \nmid n_{i}$. Finiteness of $G$ allows us to treat a projective module as injective, and vice versa (see, [1, Theorem (52.3), p. 421]). From (2.5), we have

Lemma 3.2. Suppose that $p \nmid n_{i}, 1 \leqq i \leqq \delta$. Then

$$
\hat{S} \bigoplus_{i=1}^{\delta} T_{i}^{G} \cong \hat{R}
$$

For a module $V$, we define $\phi V$ to be the smallest submodule of $V$ such that $V / \phi V$ is completely reducible. Equivalently, $\phi V$ is the intersection of all maximal submodules of $V$. We say that $p$ is semi-coprime to $n$ (with respect to $X$ ) if $p \mid n$ but $p \nmid n_{i}, 1 \leqq i \leqq \delta$. Gaschütz's theorem ([4, Theorem 2.9, p. 9]) and Lemma (3.2) yield:

Proposition 3.3. Suppose that $p$ is semi-coprime to $n$. Let

$$
0 \rightarrow J \rightarrow V \stackrel{f}{\rightarrow} W \stackrel{g}{\rightarrow} \mathbb{F}_{p} \rightarrow 0
$$

be an $\mathbb{F}_{p} G$-exact sequence, where $V$ and $W$ are projective modules, with $\operatorname{Ker} g=\phi W$ and $J \leqq \phi V$. Then

$$
\hat{S} \oplus V \oplus \mathbb{F}_{p} G \cong J \bigoplus_{i=1}^{\delta} \mathbf{g}_{i}^{G} \bigoplus_{i=\delta+1}^{d} b_{i} \mathbb{F}_{p} G \oplus W,
$$

and $J$ is a non-zero, non-projective and indecomposable module.

The Krull-Schmidt theorem may be applied to simplify the isomorphism of (3.1) and (3.3) in various cases. For example, when $\delta=0$, Proposition (3.1) and (3.3) reduce to Gaschütz's theorem ([4, Theorem 2.7 , p. 8 , Theorem 2.9 , p. 9]).

Of course, the non-projective part $N(\hat{S})$ of $\hat{S}$ in the semi-coprime case is non-zero and indecomposable, as well as isomorphic to $N(\hat{R})$. None of these properties of $N(\hat{S})$ hold for the non semi-coprime case.

(It should be noted that, in all the examples of this and the next section, we consider 
only those relative relation modules, the middle term of whose relative relation sequences do not contain any free direct summand (that is when $\delta=d$ )).

Example 3.4. Let $G=S_{3}, p=2$ and $X=\left\{g_{1}, g_{2}\right\}$, where $g_{1}^{2}=g_{2}^{3}=1$. We know that $\mathbb{F}_{2} S_{3}$ has only two distinct principle indecomposable modules, one of them being irreducible. Then it follows that $\hat{S}$ is isomorphic to the irreducible two dimensional module and so $N(\hat{S})=\{0\}$.

Example 3.5. Let $G=S_{4}, p=3, X=\left\{g_{1}, g_{2}\right\}$, where $g_{1}^{2}=g_{2}^{3}=1$. We know (see [8, Ex. 18.5 , p. 153]) that $\mathbb{F}_{3} S_{4}$ has precisely four principal indecomposable modules each of dimension three; two of them are irreducible. The other two, using (2.9), in applying (3.3) to obtain $N(\hat{R})$, give that $\operatorname{dim} N(\hat{R})=1$. Since the dimension of $\hat{S}$ is five (see Corollary (2.6)), therefore the dimension of $N(\hat{S})$ is either two or five. In any case, $N(\hat{S})$ cannot be a homomorphic image of $N(\hat{R})$.

In view of remark (4.9) below, $N(\hat{S})$ in the non semi-coprime case can be decomposable.

A suitable characterisation of $N(\hat{S})$, and so one of $\hat{S}$ in general, remains unknown, however when $G$ is a $p$-group and $X$ is a minimal generating set for $G, \hat{S}$ turns out to be non-projective and indecomposable, which is discussed by the author in [9].

\section{Comparison of relative relation modules}

Let $X_{1}=\left\{g_{i}, 1 \leqq i \leqq d_{1}\right\}$ and $X_{2}=\left\{h_{i}, 1 \leqq i \leqq d_{2}\right)$ be two generating sets of $G$, and consider the resulting relative relation sequences

$$
0 \rightarrow \hat{S}_{1} \rightarrow \bigoplus_{i=1}^{\delta_{1}} \mathbf{g}_{i}^{G} \underset{i=\delta_{1}+1}{d_{1}} b_{i} \mathbb{F}_{p} G \stackrel{\varphi_{1}}{\rightarrow} \mathbf{g} \rightarrow 0 .
$$

and

$$
0 \rightarrow \widehat{S}_{2} \rightarrow \bigoplus_{i=1}^{\delta_{2}} \mathbf{h}_{i}^{G} \bigoplus_{i=\delta_{2}+1}^{d_{2}} b_{i} \mathbb{F}_{p} G \stackrel{\Phi_{2}}{\rightarrow} \mathbf{g} \rightarrow 0
$$

where $\hat{S}_{i}=S_{i} / S_{i}^{\prime} S_{i}^{p}, i=1,2$. Whenever $\delta_{i}=0$, we shall identify $\hat{S}_{i}$ with $\hat{R}_{i}$.

An application of Schanuel's Lemma ([3, Lemma 11, p. 162]) to (4.1) and (4.2), together with the Krull-Schmidt theorem on putting $\delta_{1}=\delta_{2}=0$ gives:

Proposition 4.3 (Gaschütz [3]). If $d_{1} \leqq d_{2}$, then $\hat{R}_{2} \cong \hat{R}_{1} \oplus L$, where $L$ is a free module of rank $d_{2}-d_{1}$.

The analogous result for relative relation modules is far from being true, which we analyse in this section. The following example shows that $\hat{S}_{1}$ and $\hat{S}_{2}$ may not be isomorphic even if there is an automorphism of $G$ mapping $X_{1}$ onto $X_{2}$. (Recall that in all examples we suppose that $\delta=d$ ). 
Example 4.4. Let $G=\mathbb{Z}_{q} \times \mathbb{Z}_{q}$, where $q$ is an integer greater than one,

$$
X_{1}=\left\{g_{1}, g_{2}\right\}, X_{2}=\left\{g_{0}, g_{2}\right\} \text {, where } g_{0}=g_{1} g_{2} \text {. }
$$

Case (i). płq. By Lemma (3.2) and Proposition (4.3)

$$
\hat{S}_{1} \oplus T_{1}^{G} \oplus T_{2}^{G} \cong \hat{S}_{2} \oplus T_{0}^{G} \oplus T_{2}^{G}
$$

Since the action of $\left\langle g_{1}\right\rangle$ is trivial on $T_{1}^{G}$ but non-trivial on $T_{0}^{G}, \hat{S}_{1}$ and $\hat{S}_{2}$ cannot be isomorphic as $G$-modules. As both $\hat{S}_{1}$ and $\hat{S}_{2}$ are projective, we also conclude that under the hypotheses the projective parts of two relative relation modules may not be isomorphic.

Case (ii) $p=q>2$. Let $x=\left(b_{1}-b_{2}\right)\left(g_{1}-1\right)\left(g_{2}-1\right)$ and $y=\left(b_{0}-b_{2}\right)\left(g_{0}-1\right)\left(g_{2}-1\right)$. It can be shown that $\hat{S}_{1}$ as an $\mathbb{F}_{p} G$-module is generated by $x$, and $\hat{S}_{2}$ by $y$. If there exists any $\mathbb{F}_{p} G$ isomorphism, $\theta$ say, of $\hat{S}_{1}$ to $\hat{S}_{2}$, then $x \theta=y a$, for some $a \in \mathbb{F}_{p} G$. As $a$ can be expressed as a sum of an element of $\mathbf{g}$ (which is generated by $\left.\left\{\left(g_{1}-1\right),\left(g_{2}-1\right)\right\}\right)$ and an element of $\mathbb{F}_{p}$, we have

$$
y a=\alpha y+y\left(g_{1}-1\right) a_{1}+y\left(g_{2}-1\right) a_{2}, \quad \alpha \in \mathbb{F}_{p}, a_{1}, a_{2} \in \mathbb{F}_{p} G .
$$

Since ya must be a generator of $\hat{S}_{2}$, we must have $\alpha \neq 0$. If $b=\left(g_{1}-1\right)^{p-1}\left(g_{2}-1\right)^{p-3}$, then using the commutativity of $\mathbb{F}_{p} G$,

$$
\begin{aligned}
(x b) \theta & =\alpha y b \quad\left(\text { as }\left(g_{1}-1\right)^{p}=0\right) \\
& =\alpha\left(b_{0}-b_{2}\right)\left(g_{1}-1\right)^{p-1}\left(g_{2}-1\right)^{p-1},
\end{aligned}
$$

which is a non-zero element. But $x b=0$, and so $\theta$ cannot be an isomorphism. It may also be noted that both $\hat{S}_{1}$ and $\hat{S}_{2}$ are non-projective (and indecomposable), as (by (2.13)) both are embedded in the unique principal indecomposable module $\mathbb{F}_{p}\left(\mathbb{Z}_{p} \times \mathbb{Z}_{p}\right)$. Thus the non-projective parts of two relative relation modules may not be isomorphic as well.

We have:

Proposition 4.5. Suppose that $d_{1}=d_{2}=d$ and $\delta_{1}=\delta_{2}=\delta$. Let $x$ be a fixed element of $G$ such that $\left\langle h_{i}\right\rangle=\left\langle g_{i}\right\rangle^{x}, 1 \leqq i \leqq \delta$. Then $\hat{S}_{1} \cong \hat{S}_{2}$.

Proof. Clearly $h_{i}=\left(g_{i}^{\alpha_{i}}\right)^{x}$, for some integer $\alpha_{i}$ such that $\left\langle g_{i}^{\alpha_{i}}\right\rangle=G_{i}, 1 \leqq i \leqq \delta$. Therefore, it suffices to prove the result in two cases, namely

(i) When $h_{i}=g_{i}^{\alpha_{i}}, 1 \leqq i \leqq \delta$, and (ii) when $h_{i}=g_{i}^{x}, 1 \leqq i \leqq \delta$.

Case (i). Clearly $\mathbf{h}_{i}^{G}=\mathbf{g}_{i}^{G}, 1 \leqq i \leqq \delta$; and so it is enough to show that $\hat{\psi}_{1}$ and $\hat{\psi}_{2}$ coincide on $h_{i}^{G}$. For, if $a_{i} \in \mathbb{F}_{p} G$,

$$
\left(b_{i}\left(h_{i}-1\right)\right) \hat{\psi}_{2}=\sum_{\gamma_{i}=0}^{a_{i}-1}\left(b_{i}\left(g_{i}-1\right) g \eta^{i}\right) a_{i}=\left(b_{i}\left(h_{i}-1\right) a_{i}\right) \hat{\psi}_{1}
$$


and so, $\widehat{S}_{1} \cong \widehat{S}_{2}$.

Case (ii). Let $\Lambda$ be the $\mathbb{F}_{p} G$-isomorphism of $\mathbb{F}_{p} G$ given by $a \rightarrow x a$, and consider

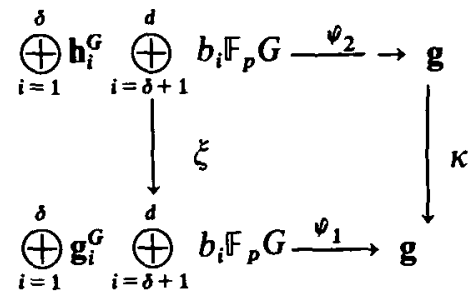

where $\kappa$ is the restriction of $\Lambda$ given by $\left(h_{i}-1\right) \rightarrow\left(g_{i}-1\right) x, 1 \leqq i \leqq d$; and $\xi$ is so defined that its restriction to $h_{i}^{G}$ and $b_{i} \mathbb{F}_{p} G$ coincide with the restriction of $\Lambda$ given by $b_{i}\left(h_{i}-1\right) \rightarrow b_{i}\left(g_{i}-1\right) x$ and $b_{i} \rightarrow b_{i} x$, respectively. Then $\xi \hat{\psi}_{1}=\hat{\psi}_{2} \kappa$, and so the existence of an isomorphism of $\hat{S}_{2}$ to $\hat{S}_{1}$, namely the restriction of $\xi$, is immediate.

It is not known whether there is an isomorphism between $\hat{S}_{1}$ and $\hat{S}_{2}$ if we only suppose that the elements of $X_{1}$ and $X_{2}$ are pairwise conjugate to each other. However, this is true in the semi-coprime case and follows from Schanuel's Lemma because the middle terms of (4.1) and (4.2) become projective.

For the rest of the section, suppose that $X_{2}=X_{1} \cup\left\{g_{0}\right\}, \delta_{2}=\delta_{1}+1$. We prove:

Lemma 4.6. $\hat{S}_{1}$ is a submodule of $\hat{S}_{2}$, and $\hat{S}_{2} / \hat{S}_{1} \cong \mathbf{g}_{0}^{G}$.

Proof. Let $V=\bigoplus_{i=1}^{\delta_{1}} \mathbf{g}_{i}^{G} \bigoplus_{i=\delta_{1}+1}^{d_{1}} b_{i} \mathbb{F}_{p} G$, and $W=\mathbf{g}_{i}^{G}$. Clearly, $\hat{\psi}_{1}$ and the restriction of $\hat{\psi}_{2}$ to $V$ coincide; and so, $S_{2} \cap V=\hat{S}_{1}$. Moreover,

$$
\left(\hat{S}_{2} / \hat{S}_{1}\right) \cap\left(V / \hat{S}_{1}\right)=\left(\hat{S}_{2} \cap V\right) / \hat{S}_{1}=\{0\}
$$

therefore the sum of $\hat{S}_{2} / \hat{S}_{1}$ and $V / \hat{S}_{1}$ is a direct sum. Since $\operatorname{dim}\left(\hat{S}_{2} / \hat{S}_{1}\right)+\operatorname{dim}\left(V / \hat{S}_{1}\right)=$ $\operatorname{dim}\left((V \oplus W) / \hat{S}_{1}\right) ;$ therefore

$$
\hat{S}_{2} / \widehat{S}_{1} \oplus V / \widehat{S}_{1}=(V \oplus W) / \widehat{S}_{1} \cong V / \widehat{S}_{1} \oplus W
$$

The Krull-Schmidt theorem now gives the required result.

The following example shows that $\hat{S}_{2}$ need not split over $\hat{S}_{1}$.

Example 4.7. Let $G=\mathbb{Z}_{2} \times \mathbb{Z}_{2}, \quad p=2, \quad X_{1}=\left\{g_{1}, g_{2}\right\}$ and $X_{2}=\left\{g_{0}, g_{1}, g_{2}\right\}$ (where $\left.g_{0}=g_{1} g_{2}\right)$.

Note that $b_{0}\left(g_{0}+1\right) g_{1}=b_{0}\left(g_{0}+1\right) g_{2}=b_{0}\left(g_{1}+g_{2}\right)$, is an element of $g_{0}^{G}$. Let $y_{1}=$ $b_{1}\left(g_{1}+g_{2}\right)+b_{1}\left(g_{1}+1\right)+b_{2}\left(g_{2}+1\right) ; \quad y_{2}=\left(b_{1}+b_{2}\right)\left(g_{1}+1\right)\left(g_{2}+1\right) \quad$ and $y_{3}=$ $b_{0}\left(g_{1}+g_{2}\right)\left(g_{1}+1\right)+b_{1}\left(g_{1}+1\right)\left(g_{2}+1\right)$. It may be verified that $y_{1}, y_{2}, y_{3}$ is an $\mathbb{F}_{p}$-linearly independent subset of $\hat{S}_{2}$, and so must be a basis, because $\operatorname{dim} \hat{S}_{2}=3$. It is easy to check that the space spanned by $y_{2}, y_{3}$ is the (unique) maximal submodule of $\hat{S}_{2}$, and so $\hat{S}_{2}$ must be indecomposable.

Finally we prove: 
Proposition 4.8. Suppose that either $g_{0} \in X_{1}$ or else $p$ does not divide the order of $\left\langle g_{0}\right\rangle$. Then $\hat{S}_{2} \cong \hat{S}_{1} \oplus \mathbf{g}_{0}^{G}$.

Proof. Suppose that $g_{0} \in X_{1}$. We suppose without loss of generality that $g_{0}=g_{1}$. Let $V=\bigoplus_{i=1}^{\delta} \mathbf{g}_{i}^{G} \bigoplus_{i=\delta+1}^{d} b_{i} \mathbb{F}_{p} G$. Let $\eta_{1}$ be the identity isomorphism of $V$, and $\eta_{2}$ the epimorphism of $V \oplus \mathbf{g}_{0}^{G}$ to $V$ such that the restriction of $\eta_{2}$ to $V$ and $\mathbf{g}_{0}^{G}$ is the identity isomorphism. Then $\hat{\psi}_{1}=\eta_{1} \hat{\psi}_{2}$ and $\hat{\psi}_{2}=\eta_{2} \hat{\psi}_{1}$. Now as in the proof of Schanuel's Lemma ([3, Lemma 11, p. 162]), we may deduce the result by applying Krull-Schmidt theorem.

When $p \nmid\left|\left\langle g_{0}\right\rangle\right|$, the result is obvious because $\mathbf{g}_{0}^{G}$ is projective.

It is not known whether $\hat{S}_{2}$ splits over $\hat{S}_{1}$ and $g_{0}^{G}$ if we only suppose that $g_{0}$ is a conjugate of an element of $X_{1}$ in $G$.

Remark 4.9. If in Proposition 4.8 we suppose that $G$ is a $p$-group and $g_{0} \in X_{1}$, then $N\left(\hat{S}_{2}\right) \cong N\left(\hat{S}_{1}\right) \oplus \mathbf{g}_{0}^{G}$, because $\mathbf{g}_{0}^{G}$ being embedded in $\mathbb{F}_{p} G$, is non-projective. This substantiates our earlier claim that the non-projective part of a relative relation module can be decomposable.

Acknowledgement. I would like to thank John Cossey and L. G. Kovacs of the Australian National University for their encouragement and valuable advice during the course of this work. Finally, I thank the referee for his valuable comments and suggestions.

\section{REFERENCES}

1. C. W. CURTIS and I. Reiner, Representation Theory of Finite Groups and Associative Algebras (Pure and Appl. Math. 11, Interscience, New York, London and Sydney, 1962).

2. W. Gaschutz, Über modulare Darstellungen endlicher Gruppen, die von freien Gruppen induziert werden, Math. Z. 60 (1954), 274-286.

3. K. W. Gruenderg, Cohomological Topics in Group Theory (Lecture notes in Mathematics 143, Springer-Verlag, Berlin, Heidelberg and New York, 1970).

4. K. W. Gruenberg, Relation Modules of Finite Groups (Regional Conference Series in Math. Number 25, Amer. Math. Soc. Providence, R.I., 1976).

5. B. Hartley and A. I. Lichtman, Relative higher relation modules, J. Pure Appl. Algebra 22 (1981), 75-89.

6. W. Magnus, On the theorem of Marshall Hall, Ann. of Math. (2) 40 (1939), 764-768.

7. W. Magnus, A. Karrass and D. Soltar, Combinatorial Group Theory (Pure and Appl. Math. 13, Interscience, New York, London and Sydney, 1966).

8. J. P. Serre, Linear Representations of Finite Groups (Graduate Texts in Mathematics 42, Springer-Verlag, New York, Heidelberg and Berlin, 1977).

9. M. Yamin, Minimal relative relation modules of finite p-groups, Proc. Amer. Math. Soc., to appear.

Department of Mathematics

Jamia Millia Islamia

New Delhi-110025

INDIA 